\title{
Trisomy 7 mosaicism, maternal uniparental heterodisomy 7 and Hirschsprung's disease in a child with Silver-Russell syndrome
}

\author{
Elisabeth Flori ${ }^{*}, 1$,Emmanuelle Girodon ${ }^{2}$, Brigitte Samama ${ }^{3}$, François Becmeur ${ }^{4}$, \\ Brigitte Viville ${ }^{5}$, Françoise Girard-Lemaire ${ }^{1}$, Bérénice Doray ${ }^{1}$, Caroline Schluth ${ }^{1}$, \\ Luc Marcellin ${ }^{6}$, Nelly Boehm ${ }^{3}$, Michel Goossens ${ }^{2}$ and Véronique Pingault ${ }^{2}$
}

\begin{abstract}
${ }^{1}$ Service de Cytogénétique, Fédération de Génétique, Hôpitaux Universitaires de Strasbourg, Strasbourg, France; ${ }^{2}$ Service de Biochimie et de Génétique, Hôpital Henri Mondor, Créteil, France; ${ }^{3}$ Institut d'Histologie, Faculté de Médecine, Université Louis Pasteur, Strasbourg, France; ${ }^{4}$ Service de Chirurgie Infantile, Hôpitaux Universitaires de Strasbourg, Strasbourg, France; ${ }^{5}$ Département de Gynécologie-Obstétrique, Hôpitaux Universitaires de Strasbourg, Strasbourg, France; ${ }^{6}$ Service d'Anatomie Pathologique, Hôpitaux Universitaires de Strasbourg, Strasbourg, France
\end{abstract}

Prenatal trisomy 7 is usually a cell culture artifact in amniocytes with normal diploid karyotype at birth and normal fetal outcome. In the same way, true prenatal trisomy 7 mosaicism usually results in a normal child except when trisomic cells persist after birth or when trisomy rescue leads to maternal uniparental disomy, which is responsible for $5.5-7 \%$ of patients with Silver-Russell syndrome (SRS). We report here on the unusual association of SRS and Hirschsprung's disease (HSCR) in a patient with maternal uniparental heterodisomy 7 and trisomy 7 mosaicism in intestine and skin fibroblasts. HSCR may be fortuitous given its frequency, multifactorial inheritance and genetic heterogeneity. However, the presence of the trisomy 7 mosaicism in intestine as well as in skin fibroblasts suggests that SRS and HSCR might possibly be related. Such an association might result from either an increased dosage of a nonimprinted gene due to trisomy 7 mosaicism in skin fibroblasts (leading to SRS) and in intestine (leading to HSCR), or from an overexpression, through genomic imprinting, of maternally expressed imprinted allele(s) in skin fibroblasts and intestine or from a combination of trisomy 7 mosaicism and genomic imprinting. This report suggests that the SRS phenotype observed in maternal uniparental disomy 7 (mUPD(7)) patients might also result from an undetected low level of trisomy 7 mosaicism. In order to validate this hypothesis, we propose to perform a conventional and molecular cytogenetic analysis in different tissues every time mUPD(7) is displayed. European Journal of Human Genetics (2005) 13, 1013-1018. doi:10.1038/sj.ejhg.5201442;

published online 25 May 2005

Keywords: uniparental disomy; Silver-Russell syndrome; Hirschsprung's disease; trisomy 7; chromosome 7

Introduction

Trisomy 7 is extremely rare at birth and is generally considered lethal in embryogenesis. ${ }^{1}$ All surviving children

*Correspondence: Dr E Flori, Service de Cytogénétique, Hôpital de Hautepierre, Avenue Molière, 67098 Strasbourg Cedex, France.

Tel: + 333881273 38; Fax: + 333881273 37;

E-mail: Elisabeth.Flori@chru-strasbourg.fr

Received 14 October 2004; revised 14 April 2005; accepted 15 April 2005; published online 25 May 2005 are mosaics with variable and nonspecific clinical features. $^{1-5}$ Chromosomal mosaicism may be suggested by body asymmetry and/or Blaschkolinear skin pigmentary dysplasia associated with developmental delay. Cultured skin fibroblasts cytogenetic analysis confirms mosaicism and identifies its chromosomal origin. In chorionic villi, trisomy 7 is usually confined placental mosaicism (CPM) and outcome is normal without intrauterine fetal growth retardation (IUGR) as observed in other CPMs. ${ }^{1,6-9}$ In 
amniocytes, trisomy 7 is frequently a cell culture artifact leading to single- or multiple-cell pseudomosaicism with normal fetal outcome. ${ }^{10,11}$ True trisomy 7 mosaicism was first reviewed by Hsu et al, ${ }^{12}$ who analysed eight cases. At term, only one, previously reported, ${ }^{3}$ was abnormal with mild developmental delay, facial asymmetry, hypomelanosis of Ito and trisomy 7 mosaicism in skin fibroblasts. Later on, two other cases were reported. In the first case, ${ }^{13}$ trisomy 7 was present in $7 / 9$ colonies (77\%) analysed from short amniocyte cultures but not in fetal blood. At the age of 3 years, the child showed asymmetric face, pigmentary changes of the skin, dysmorphic facial features and normal development. Trisomy 7 mosaicism was confirmed in two skin fibroblast cultures (24 and 36\%). Maternal uniparental disomy (mUPD) for chromosome 7 was not investigated but no growth retardation was noted at 3 years 6 months (height was $97 \mathrm{~cm}$ ). In the second case, ${ }^{14}$ trisomy 7 was found in 13 out of 32 colonies studied from amniocyte cultures (41\%). DNA polymorphism analysis revealed that the diploid cell line occurred through a trisomy rescue event and resulted in maternal uniparental heterodisomy with both chromosomes 7 originating from the mother. At birth, the child was small for gestational age and displayed some of the clinical features observed in SRS. Placental cytogenetic analysis revealed nonmosaic trisomy 7 and cord blood cells were all disomic. No cytogenetic analysis was performed in skin fibroblasts.

$\operatorname{mUPD}(7)$ is considered to be responsible for approximately $5.5-7 \%$ of patients with the Silver-Russell syndrome (SRS) phenotype. ${ }^{15-22}$ SRS includes severe IUGR, postnatal short stature, retarded bone age, relative macrocephaly, triangular face, prominent and bossed forehead, small chin, micrognathia, craniofacial disproportion, down-turned mouth corners, brachydactyly and clinodactyly of fifth fingers, variable asymmetry of the face, body and limbs and café-au-lait spots. MUPD(7) patients display most of the same features. ${ }^{19}$ However, a wide mouth with down-turned corners, prominent ears, café-au-lait spots, hemihypertrophy and clinodactyly of fifth fingers seem to be more rarely present; ${ }^{19}$ moreover, their chin is pointed and they demonstrate specific symptoms such as speech delay, severe feeding difficulties in the first years of life and excessive sweating. ${ }^{21}$ UPD refers to inheritance of homologous chromosomes from only one parent; it results either in heterodisomy when the two homologs are different or isodisomy when they are two copies of the same chromosome. Heterodisomy implies parental meiosis nondisjunction. Isodisomy may result from mitotic duplication in a monosomic zygote or from chromosome loss of a trisomic zygote originating from a postzygotic segregation error. UPD may be either a complete disomy of an entire chromosome or a mixed disomy with combination of isoand heterodisomy due to meiotic recombination events. ${ }^{17}$ Both maternal hetero- and isodisomy for chromosome 7 have been associated with the same SRS phenotype. ${ }^{16-23}$
Hirschsprung's disease (HSCR) or congenital aganglionic megacolon is a frequent cause of congenital intestinal obstruction. HSCR is a developmental disorder characterized by the absence of intramural ganglion cells of the myenteric Auerbach's and the submucosal Meissner's plexuses. HSCR occurs as an isolated trait in 70\% of cases and is associated with a chromosomal abnormality, especially trisomy 21 , in $12 \%$ of cases and with other congenital anomalies in $18 \%$ of patients. ${ }^{24}$ To our knowledge, to date, no SRS or $\operatorname{mUPD}(7)$ has been reported in association with HSCR. We report here on an SRS patient with trisomy 7 mosaicism, mUPD(7) and HSCR and discuss their possible relationships.

\section{Clinical report}

During her first pregnancy, a 26-year-old woman was referred to the gynaecology unit at 22 weeks of gestation because of prenatal sonographic detection of a single umbilical artery. The parents were healthy and not related and there was no family history of congenital malformations. A detailed level II ultrasound examination showed moderate oligoamnios and suspected IUGR. An amniocentesis was performed and revealed a male karyotype with trisomy 7 mosaicism. No search for UPD was performed because the father refused blood sampling at that time. Genetic counselling was given taking into account trisomy 7 mosaicism and possible mUPD(7) because of IUGR. As a subsequent sonographic examination failed to find IUGR, the parents elected to continue the pregnancy. Induced delivery due to premature rupture of membranes occurred at 35 weeks of gestation. After brief ventilation, the boy was in good health (Apgar scores X/IX/X). Length $(42 \mathrm{~cm})$, weight $(1900 \mathrm{~g})$ and head circumference (OFC) $(31 \mathrm{~cm})$ were within the normal range. Physical examination displayed some minor abnormalities: prominent large forehead, prominent nasal bridge, low posterior-rotated ears, small and retruded chin, bilateral clinodactyly of fifth fingers and bilateral simian creases. Genitalia were normal. Cardiac ultrasound showed ventricular septal defect. X-ray skeletal survey was normal except clinodactyly of the fifth fingers. Transfontanellar and abdominal ultrasounds showed no anomaly.

At 6 months, the child was admitted to hospital for bronchitis. During the hospitalization, he once had vomiting and hard and bloody stools. At 13 months, postnatal growth retardation became obvious with weight of $6 \mathrm{~kg}(-4 \mathrm{SD})$ and length of $67 \mathrm{~cm}(-3 \mathrm{SD})$ and there was a relative macrocephaly with normal OFC $(47 \mathrm{~cm})$. The child had feeding difficulties and did not eat solids. He displayed global hypotonia, did not sit, began only to transfer objects from one hand to the other and required physiotherapy. By contrast, his development was normal in fine motor control and social skills. Facial dysmorphy included a 
small triangular-shaped face with frontal bosses and narrow palpebral fissures, a small mouth with thin lips, a pointed chin and low posterior-rotated and overfolded ears. Because of repetitive bile vomiting, severe constipation and chronic abdominal distension, the child was readmitted and several rectal biopsies were performed. They demonstrated alternately a paucity or absence of intramural ganglion cells of myenteric and submucosal plexuses in colic mucosa as well as in muscularis and ascertained the existence of HSCR. A one-stage surgical treatment could be performed with colono-anal anastomosis by a transanal approach; no short-term complications were observed. A rectal biopsy was performed during surgery for cell culture and cytogenetic analyses.

At 24 months, the child stood with support. Social skills, behavioural concerns and receptive speech were within the normal range. He had learning disability in expressive speech; he did not yet eat solids and had poor weight gain. Speech therapy was initiated in association with psychomotricity and special education training.

At 30 months, he walked alone. Height was $77 \mathrm{~cm}(-4$ SD), weight $8100 \mathrm{~g}$ ( $-4 \mathrm{SD}$ ) and OFC $49 \mathrm{~cm}$ (normal range). Bone age was delayed. Endocrinology evaluation and growth hormone stimulation studies were normal. Neither body asymmetry nor pigmentary abnormalities were observed.

At 3 years, the child expressed himself by signs and began to communicate by expressive language. At 5 years, facial dysmorphy persisted (Figure 1) and growth hormone treatment, introduced at 3 years, showed good short-term results with height at $105 \mathrm{~cm}(-1.5 \mathrm{SD})$.

\section{Conventional cytogenetic investigations (Table 1)}

Prenatal studies Short-term in situ amniocyte cultures from four coverslips were set up and 18 colonies were analysed. Karyotype was normal $46, \mathrm{XY}$ in $10 / 18$ colonies and detected a trisomy 7 in $8 / 18$ colonies, demonstrating true trisomy 7 mosaicism (44\%).
Postnatal studies At birth, trisomy 7 mosaicism was also found in $79 \%$ of cells in term placenta cultures, but the karyotype was normal, 46,XY, in blood lymphocytes as well as in fibroblast cultures from the umbilical cord. At 1 year, analysis of fibroblast cultures from the rectal biopsy found trisomy 7 mosaicism in 15 mitoses of the 100 analysed. At 3 years, chromosome analysis performed in cultured skin fibroblasts of the child in order to compare it with the result obtained from the rectal biopsy was normal.

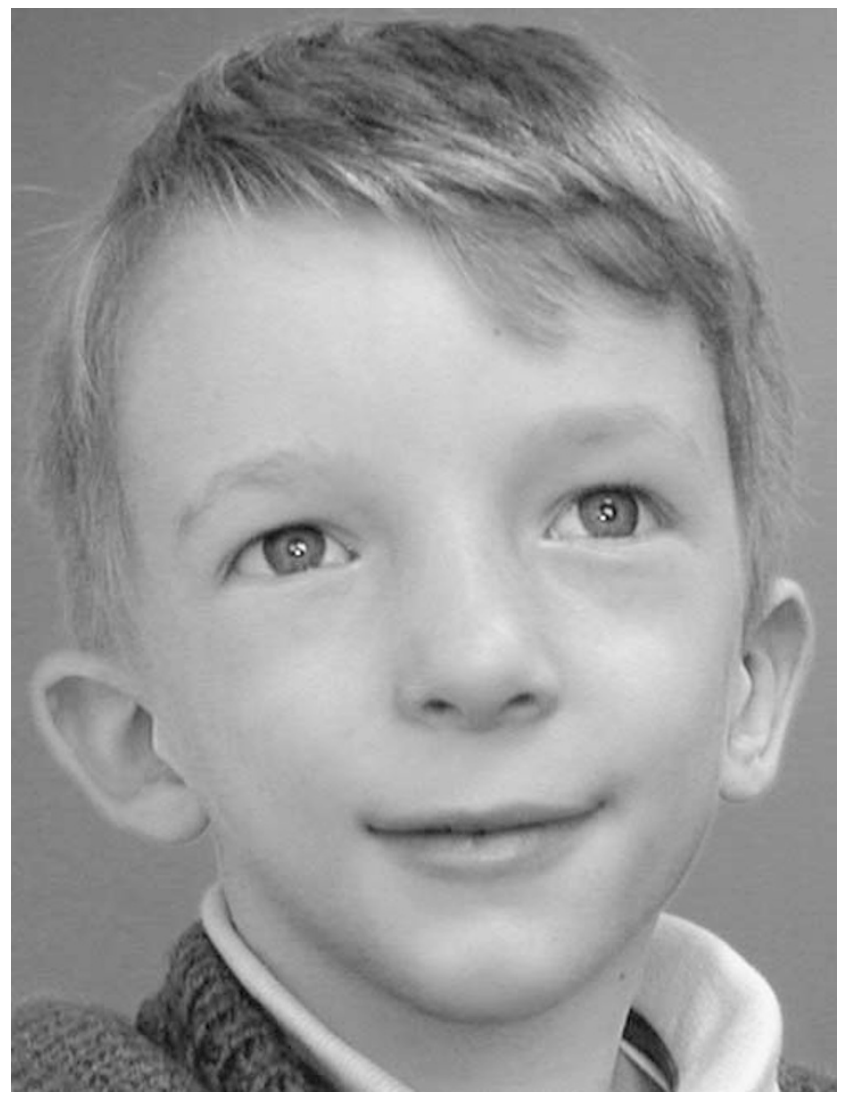

Figure 1 The patient at 5 years.

Table 1 Prenatal and postnatal cytogenetic studies

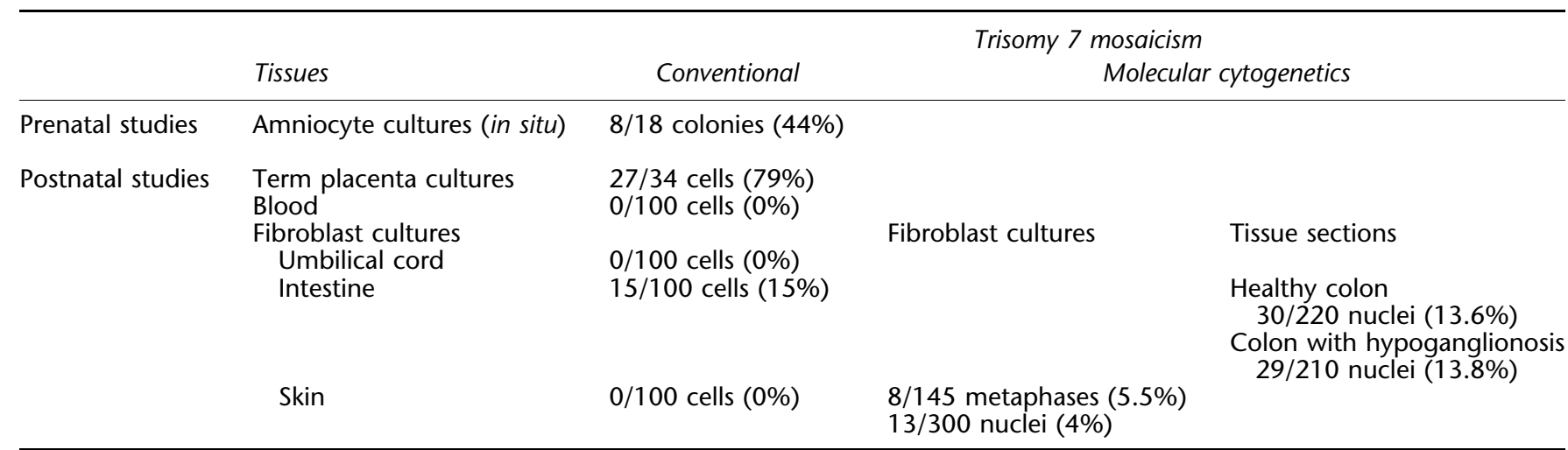




\section{Molecular cytogenetic studies (Table 1)}

Fluorescent in situ hybridization with a chromosome 7 alpha-satellite DNA probe from Oncor (D7Z1) was performed in skin fibroblast interphase nuclei and on metaphase chromosomes. Trisomy 7 mosaicism was detected in 4 and $5.5 \%$, respectively.

Paraffin-embedded tissue sections of two different surgical resected colon fragments were processed by fluorescent in situ hybridization: one fragment corresponded to a healthy zone and the other showed hypoganglionosis. Double labelling with chromosome 7 and chromosome 17 alpha-satellite DNA probes was performed in order to detect trisomy 7 mosaicism. Simultaneous labelling with a chromosome 17 probe served as an internal hybridization control to eliminate a possible polyploidy. Trisomy 7 mosaicism was detected at the same rate on the healthy colon fragment $(13.6 \%)$ and on the fragment with hypoganglionosis (13.8\%). The analysed nuclei with trisomy 7 were not only localized in nervous plexuses but also in the epithelium and connective and muscular tissues.

\section{Molecular studies (Table 2)}

A microsatellite analysis of chromosome 7 was performed using the linkage mapping set (Applied Biosystem). A total of 10 markers located along the whole chromosome 7 were analysed on DNA extracted from blood samples, according to the manufacturer's protocol. For five markers (D7S531, D7S630, D7S486, D7S530, D7S640), the father shared no allele with his son, so no chromosome 7 was of paternal origin. For the 10 analysed microsatellites, alleles were the same for the child and his mother at the heterozygous state (eight markers) as well at the homozygous one (two markers). Therefore, the child presented with a complete chromosome 7 heterodisomy in blood.

Table 2 Microsatellite analysis

\begin{tabular}{lllll}
\hline & & Mother & Son & Father \\
\hline D75531 & $7 \mathrm{p} 22$ & $\mathrm{~B} / \mathrm{B}$ & $\mathrm{B} / \mathrm{B}$ & $\mathrm{A} / \mathrm{C}$ \\
D7S493 & $7 \mathrm{p} 21$ & $\mathrm{~A} / \mathrm{C}$ & $\mathrm{A} / \mathrm{C}$ & $\mathrm{A} / \mathrm{B}$ \\
D7S519 & $7 \mathrm{p} 11.2$ & $\mathrm{~A} / \mathrm{B}$ & $\mathrm{A} / \mathrm{B}$ & $\mathrm{A} / \mathrm{C}$ \\
D75630 & $7 \mathrm{q} 21.1$ & $\mathrm{~A} / \mathrm{B}$ & $\mathrm{A} / \mathrm{B}$ & $\mathrm{C} / \mathrm{D}$ \\
D75657 & $7 \mathrm{q} 21.1$ & $\mathrm{~B} / \mathrm{C}$ & $\mathrm{B} / \mathrm{C}$ & $\mathrm{A} / \mathrm{B}$ \\
D7S515 & $7 \mathrm{q} 21.3-\mathrm{q} 22.1$ & $\mathrm{~B} / \mathrm{C}$ & $\mathrm{B} / \mathrm{C}$ & $\mathrm{A} / \mathrm{B}$ \\
D7S486 & $7 \mathrm{q} 31$ & $\mathrm{~B} / \mathrm{B}$ & $\mathrm{B} / \mathrm{B}$ & $\mathrm{A} / \mathrm{C}$ \\
D7S530 & $7 \mathrm{q} 32$ & $\mathrm{C} / \mathrm{D}$ & $\mathrm{C} / \mathrm{D}$ & $\mathrm{A} / \mathrm{B}$ \\
D7S640 & $7 \mathrm{q} 32-33$ & B/D & B/D & A/C \\
D7S661 & $7 \mathrm{q} 35-\mathrm{q} 36$ & A/B & A/B & B/B \\
\hline
\end{tabular}

The microsatellites are ranged according to their location along the chromosome and the alleles are named by size order. The microsatellites allowing exclusion of the transmission of a paternal chromosome are indicated in bold.

\section{Discussion}

In the reported case, true trisomy 7 mosaicism could be ascertained in amniocyte cultures, as trisomy 7 was present in several independent primary cultures ${ }^{11}$ as well as in skin fibroblasts at the age of 3 years. The absence of clinical chromosomal mosaicism markers may be explained by the low percentage of trisomic 7 cells in skin fibroblasts. By contrast, the phenotype of the patient was compatible with SRS associated with mUPD(7). The most likely mechanism of $\operatorname{mUPD}(7)$ in the present case is a trisomic zygote rescue. Given the maternal complete heterodisomy of chromosome 7 observed with molecular studies, it can be assumed that a trisomic zygote originated by maternal nondisjunction at the first meiotic cell division. Postzygotic loss of one supernumerary chromosome 7 restored a diploid fetal karyotype. As previously demonstrated, disruption of genomic imprinting is the most likely cause for SRS either by absence of the active paternal allele involved in growth promotion or by excess of the maternal allele involved in growth inhibition. ${ }^{20}$ Overexpression of the maternal allele, previously hypothesized in two SRS non-mUPD(7) patients with duplication of $7 \mathrm{p} 12.1-\mathrm{p} 13^{25}$ and $7 \mathrm{p} 11.2-\mathrm{p} 13,{ }^{26}$ was recently confirmed in one of them by a real-time PCR assay. ${ }^{27}$ It has been suggested that two separate candidate regions, 7p11.2-p13 and 7q31-qter, are involved in patients with SRS through genomic imprinting. In the $7 p$ region, the growth factor receptor-bound protein 10 (GRB10) has been proposed as a candidate gene for SRS on the basis of its function as a growth inhibitor and its imprinting status in the mouse. However, absence of mutations in GRB10 in a cohort of 139 SRS patients ${ }^{20,28,29}$ and absence of GRB10 imprinting in growth plate cartilage directly involved in linear growth make it unlikely that GRB10 is the gene responsible for SRS. ${ }^{30}$ Nevertheless, the chromosomal 7p11.2-p13 region remains a good candidate region for SRS since several SRS patients carrying $7 p$ rearrangements including GRB10 have been reported, ${ }^{25,26,31-33}$ or, as imprinted genes are often organized in clusters, SRS could result from overexpression of maternally imprinted genes mapped proximal and distal to GRB10. In the 7q31-qter region, two imprinted genes, PEG/MST and $\gamma 2$-COP, ${ }^{34}$ and, more recently, carboxypeptidase A genes ${ }^{35}$ have also been investigated as candidates for SRS. However, their role remains uncertain in the aetiology of SRS. It is possible that both regions cause SRS through genomic imprinting ${ }^{20}$ or that another imprinted gene is involved. Epigenetic modifications or alterations in regulatory elements could also contribute to the SRS phenotype. $^{28,29}$

Another point to emphasize in the reported case is the presence of HSCR. Among the several specific genes involved in HSCR, the RET receptor tyrosine kinase seems to be a major susceptibility gene. However, mutations in RET are identified in only $50 \%$ of familial and $15-20 \%$ of sporadic HSCR cases. ${ }^{36}$ As RET has been involved in the 
development of neural crest derivatives and HSCR in particular, it has been suggested that downstream components of the RET signal transduction pathway might also predispose to HSCR. ${ }^{37}$ GRB10 was considered as a good candidate for HSCR susceptibility according to its early expression in the embryo ${ }^{38,39}$ which is consistent with a role in enteric nervous system development. ${ }^{37}$ However, no linkage or mutations of human GRB10 have been observed in a large panel of HSCR patients analysed. ${ }^{37}$ It has been demonstrated ${ }^{28,40}$ through direct analysis of allelic transcription that the human GRB10 gene is specifically imprinted in the developing central nervous system (CNS), including brain and fetal spinal cord. Also, all but two GRB10 transcripts are exclusively paternally expressed in the fetal CNS, contrary to the mouse where GRB10 is exclusively transcribed from the maternal allele. However, no allele-specific methylation has been shown to explain this fetal brain imprinted expression. It has been hypothesized that imprinted GRB10 expression might result from promoter-specific imprinting or be controlled by neighbouring or overlapping transcripts specifically expressed in the human fetal brain and skeletal muscle. ${ }^{28}$ In the same way, one of the four GRB10 isoforms, $\gamma 1$, has been demonstrated to show maternal monoallelic expression in skeletal muscle. In other fetal tissues including vertebral column, eye, tongue, stomach, intestine, heart, skin, trophoblast, kidney, pancreas, limb, umbilical cord, yolk sac, adrenal, liver and lung, all human GRB10 variants show biallelic expression.

In the reported case, different hypotheses can be raised to explain the presence of trisomy 7 mosaicism in intestine and skin fibroblasts, mUPD(7), SRS and HSCR: (1) HSCR may be fortuitous given its frequency, multifactorial inheritance and genetic heterogeneity. (2) An increased dosage of a nonimprinted gene due to trisomy 7 mosaicism in skin fibroblasts, resulting in SRS by an excess of an allele involved in growth inhibition and, in intestine, resulting in HSCR. In the intestine, the presence of trisomy 7 in nervous plexuses as well as in the epithelium or the connective and muscular tissues could account for this second hypothesis. (3) An abnormality of genomic imprinting, with either an absence of expression of a paternally active allele in disomic cells or an overexpression of specifically maternally expressed imprinted allele(s) in skin fibroblasts and intestine. In support of the latter, overexpression of certain GRB10 isoforms has been shown to inhibit tyrosine kinase activity, resulting in growth suppression and fetal CNS development inhibition. ${ }^{28,37}$ In the present study, the absence of other anomalies of fetal CNS development could be explained either by intestinalspecific mosaicism and very low or absence of trisomy 7 mosaicism in other tissues or by tissue-specific imprinting differences. (4) SRS phenotype and HSCR could also result from trisomy 7 rescue by a combination of trisomy 7 mosaicism and genomic imprinting.
As previously hypothesized, ${ }^{8,15,20,28}$ this report suggests that the SRS phenotype observed in $\operatorname{mUPD}(7)$ patients might also result from an undetected low-level trisomy 7 mosaicism. In order to validate this hypothesis, we propose to perform an extensive conventional and molecular cytogenetic analysis not only in blood but also in skin fibroblasts every time $\operatorname{mUPD}(7)$ is observed.

\section{Acknowledgements}

We thank D Huot, S Florent, $M$ Loriot, E Schmitt and S Viciana for their technical assistance and the patient's parents for their cooperation.

\section{References}

1 Magenis E, Webb MJ, Spears B, Opitz JM: Blaschkolinear malformation syndrome in complex trisomy-7 mosaicism. Am J Med Genet 1999; 87: 375-383.

2 Hodes ME, Gleiser S, DeRosa GP et al: Trisomy 7 mosaicism and manifestations of Goldenhar syndrome with unilateral radial hypoplasia. J Craniofac Genet Dev Biol 1981; 1: 49-55.

3 Jenkins D, Martin K, Young ID: Hypomelanosis of Ito associated with mosaicism for trisomy 7 and apparent 'pseudomosaicism' at amniocentesis. J Med Genet 1993; 30: 783-784.

4 Verghese S, Newlin A, Miller M, Burton BK: Mosaic trisomy 7 in a patient with pigmentary abnormalities. Am J Med Genet 1999; 87: 371-374.

5 Kayser M, Henderson LB, Kreutzmann J, Schreck R, Graham Jr JM: Blaschkolinear skin pigmentary variation due to trisomy 7 mosaicism. Am J Med Genet 2000; 95: 281-284.

6 Kalousek DK, Howard-Peebles PN, Olson SB et al: Confirmation of CVS mosaicism in term placentae and high frequency of intrauterine growth retardation association with confined placental mosaicism. Prenat Diagn 1991; 11: 743-750.

7 Kalousek DK, Langlois S, Barrett IJ et al: Uniparental disomy for chromosome 16 in humans. Am J Hum Genet 1993; 52: 8-16.

8 Kalousek DK, Langlois S, Robinson WP et al: Trisomy 7 CVS mosaicism: pregnancy outcome, placental and DNA analysis in 14 cases. Am J Med Genet 1996; 65: 348-352.

9 Wolstenholme J: Confined placental mosaicism for trisomies 2, 3, $7,8,9,16$ and 22: their incidence, likely origins, and mechanisms for cell lineage compartmentalization. Prenat Diagn 1996; 16: 511-524.

10 Hsu LY: United States survey on chromosome mosaicism and pseudomosaicism in prenatal diagnosis. Prenat Diagn 1984; 4: 97-130.

11 Hsu LYF, Kaffe S, Jenkins EC et al: Proposed guidelines for diagnosis of chromosome mosaicism in amniocytes based on data derived from chromosome mosaicism and pseudomosaicism studies. Prenat Diagn 1992; 12: 555-573.

12 Hsu LY, Yu MT, Neu RL et al: Rare trisomy mosaicism diagnosed in amniocytes, involving an autosome other than chromosomes 13, 18, 20, and 21: karyotype/phenotype correlations. Prenat Diagn 1997; 17: 201-242.

13 Kivirikko S, Salonen R, Salo A, Von Koskull H: Prenatally detected trisomy 7 mosaicism in a dysmorphic child. Prenat Diagn 2002; 22: $541-544$.

14 Bilimoria KY, Rothenberg JM: Prenatal diagnosis of a trisomy 7/ maternal uniparental heterodisomy 7 mosaic fetus. Am J Med Genet 2003; 118A: 60-63.

15 Kotzot D, Schmitt S, Bernasconi F et al: Uniparental disomy 7 in Silver-Russell syndrome and primordial growth retardation. Hum Mol Genet 1995; 4: 583-587.

16 Eggermann T, Wollmann HA, Kuner R et al: Molecular studies in 37 Silver-Russell syndrome patients: frequency and etiology of uniparental disomy. Hum Genet 1997; 100: 415-419. 
17 Preece MA, Price SM, Davies V et al: Maternal uniparental disomy 7 in Silver-Russell syndrome. J Med Genet 1997; 34: 6-9.

18 Price SM, Stanhope R, Garrett C, Preece MA, Trembath RC: The spectrum of Silver-Russell syndrome: a clinical and molecular genetic study and new diagnostic criteria. J Med Genet 1999; 36: $837-842$

19 Kotzot D, Balmer D, Baumer A et al: Maternal uniparental disomy 7 - review and further delineation of the phenotype. Eur J Pediatr 2000; 159: 247-256.

20 Hitchins MP, Stanier P, Preece MA, Moore GE: Silver-Russell syndrome: a dissection of the genetic aetiology and candidate chromosomal regions. J Med Genet 2001; 38: 810-819.

21 Hannula K, Kere J, Pirinen S, Holmberg C, Lipsanen-Nyman M: Do patients with maternal uniparental disomy for chromosome 7 have a distinct mild Silver-Russell phenotype? J Med Genet 2001; 38: $273-278$.

22 Kotzot D: Review and meta-analysis of systematic searches for uniparental disomy (UPD) other than UPD 15. Am J Med Genet 2002; 111: 366-375

23 Langlois S, Yong SL, Wilson RD, Kwong LC, Kalousek DK: Prenatal and postnatal growth failure associated with maternal heterodisomy for chromosome 7. J Med Genet 1995; 32: 871-875.

24 Amiel J, Lyonnet S: Hirschsprung disease, associated syndromes, and genetics: a review. J Med Genet 2001; 38: 729-739.

25 Joyce CA, Sharp A, Walker JM, Bullman H, Temple IK: Duplication of 7p12.1-p13, including GRB10 and IGFBP1, in a mother and daughter with features of Silver-Russell syndrome. Hum Genet 1999; 105: 273-280.

26 Monk D, Wakeling EL, Proud V et al: Duplication of 7p11.2-p13, including GRB10, in Silver-Russell syndrome. Am J Hum Genet 2000; 66: 36-46.

27 Eggermann T, Meyer E, Wollmann HA: Quantification of GRB10 in $7 \mathrm{p} 12-\mathrm{p} 14$ by fluorogenic $5^{\prime}$ nuclease chemistry and application for genetic diagnosis in Silver-Russell syndrome. Ann Genet 2004; 47: 99-102.

28 Blagitko N, Mergenthaler S, Schulz U et al: Human GRB10 is imprinted and expressed from the paternal and maternal allele in a highly tissue- and isoform-specific fashion. Hum Mol Genet 2000; 9: 1587-1595.

29 Mergenthaler S, Hitchins MP, Blagitko-Dorfs N et al: Conflicting reports of imprinting status of human GRB1O in developing brain: how reliable are somatic cell hybrids for predicting allelic origin of expression? Am J Hum Genet 2001; 68: 543-544.
30 McCann JA, Zheng H, Islam A, Goodyer CG, Polychronakos C: Evidence against GRB1O as the gene responsible for SilverRussell syndrome. Biochem Biophys Res Commun 2001; 286: 943-948.

31 Eggerding FA, Schonberg SA, Chehab FF, Norton ME, Cox VA Epstein CJ: Uniparental isodisomy for paternal $7 p$ and maternal 7q in a child with growth retardation. Am J Hum Genet 1994; 55: $253-265$.

32 Myioshi $\mathrm{O}$, Kondoh $\mathrm{T}$, Taneda $\mathrm{H}$, Otsuka $\mathrm{K}$, Matsumoto $\mathrm{T}$, Niikawa N: 47,XX,UPD(7)mat, +r(7)pat/46,XX,UPD(7)mat mosaicism in a girl with Silver-Russell syndrome (SRS): possible exclusion of the putative SRS gene from a $7 \mathrm{p} 13-\mathrm{q} 11$ region. J Med Genet 1999; 36: 326-329.

33 Nakabayashi K, Fernandez BA, Teshima I et al: Molecular genetic studies of human chromosome 7 in Silver-Russell syndrome. Genomics 2002; 79: 186-196.

34 Hannula K, Lipsanen-Nyman M, Kontiokari T, Kere J: A narrow segment of maternal uniparental disomy of chromosome 7q31qter in Silver-Russell syndrome delimits a candidate gene region. Am J Hum Genet 2001; 68: 247-253.

35 Bentley L, Nakabayashi K, Monk D et al: The imprinted region on human chromosome $7 \mathrm{q} 32$ extends to the carboxypeptidase A gene cluster: an imprinted candidate for Silver-Russell syndrome. J Med Genet 2003; 40: 249-256.

36 Attie T, Pelet A, Edery P et al: Diversity of RET proto-oncogene mutations in familial and sporadic Hirschsprung disease. Hum Mol Genet 1995; 4: 1381-1386.

37 Angrist M, Bolk S, Bentley K, Nallasamy S, Halushka MK, Chakravarti A: Genomic structure of the gene for the $\mathrm{SH} 2$ and pleckstrin homology domain-containing protein GRB1O and evaluation of its role in Hirschsprung disease. Oncogene 1998; 17: $3065-3070$

38 Pandey A, Duan H, Di Fiore PP, Dixit VM: The Ret receptor protein tyrosine kinase associates with the SH2-containing adapter protein GRB10. J Biol Chem 1995; 270: 21461-21463.

39 Angrist M, Bolk S, Halushka M, Lapchak PA, Chakravarti A: Germline mutations in glial cell line-derived neurotrophic factor (GDNF) and RET in a Hirschsprung disease patient. Nat Genet 1996; 14: 341-344.

40 Hitchins MP, Monk D, Bell GM et al: Maternal repression of the human GRB10 gene in the developing central nervous system; evaluation of the role for GRB10 in Silver-Russell syndrome. Eur $J$ Hum Genet 2001; 9: 82-90. 